\title{
Efecto de los parámetros de maquinado, estrategia de maquinado y radio de curvatura en la calidad geométrica de superficies curvas fresadas en titanio
}

\author{
Effect of machining parameters, machining strategy and curvature radio in the \\ geometric quality of curved titanium surfaces
}

Julio Gregorio Blanco Beltran ${ }^{1 *} \quad$ Marco Antonio Velasco Peña ${ }^{2} \quad$ Carlos Julio Cortés Rodríguez ${ }^{3}$

Recibido 30 de octubre de 2018, aceptado 11 de enero de 2020

Received: October 30, 2018 Accepted: January 11, 2020

\begin{abstract}
RESUMEN
El objetivo de este estudio es determinar la influencia de la velocidad de corte, avance por diente y la estrategia de maquinado; sobre la calidad geométrica de la superficie mecanizada en el fresado con herramienta de punta esférica del titanio grado quirúrgico Ti6Al4V (en este trabajo, se considera calidad geométrica; a los parámetros de rugosidad superficial $R_{a}$, precisión dimensional y tolerancia geométrica). Las probetas se mecanizaron, por medio de fresado con herramienta de punta esférica. El análisis de la influencia de los factores se realizó por medio de un diseño experimental factorial $2^{3}$. Por otra parte, un ANOVA se realizó, entre superficies esféricas cóncavas y convexas del mismo radio; para determinar la influencia sobre la calidad geométrica de la superficie mecanizada. Adicionalmente, un ANOVA se realizó entre superficies esféricas convexas de radio diferente, para determinar la influencia sobre la calidad geométrica de la superficie mecanizada. Los resultados del diseño experimental factorial $2^{3}$ mostraron que el factor estrategia de mecanizado tiene efecto sobre el parámetro de rugosidad $R_{a}$. Por otra parte, el ANOVA para las superficies esféricas cóncavas y convexas, muestra que el cambio de superficie cóncava a convexa tiene efecto sobre la tolerancia geométrica. Por último, el ANOVA para superficies esféricas convexas muestra que el cambio de radio tiene efecto sobre los parámetros de rugosidad $R_{a}, R_{q}$ y $R_{z}$, la tolerancia de geométrica y la precisión dimensional.
\end{abstract}

Palabras clave: Superficies curvas, estrategias de maquinado, tolerancia de geométrica.

\begin{abstract}
The aim of this study is to determine the influence of the cutting speed, feed per tooth and machining strategy; on geometric quality of the machined surface, in the ball milling tool of the surgical grade titanium Ti6Al4V (In this paper, geometrical quality, is considered; parameters of surface roughness, dimensional accuracy and shape tolerance). The samples were machined by means of ball milling tool. Analysis of the influence of the factors was performed by means of a $2^{3}$ factorial experimental design. Further, an ANOVA (analysis of variance) was performed between concave and convex spherical geometries of the same radius, to determine the influence on the machined surface geometric quality geometric quality of the machined surface. Additionally, an ANOVA was performed; between convex spherical geometries different radius to determine the influence on the geometric quality of the machined surface. The factorial
\end{abstract}

1 Servicio Nacional de Aprendizaje - SENA. Centro de Diseño y Metrología. Bogotá D.C., Colombia. E-mail: jublancob@sena.edu.co

2 Universidad Distrital Francisco José de Caldas. Facultad Tecnológica Bogotá, Colombia. E-mail: mavelascop@udistrital.edu.co

3 Universidad Nacional de Colombia. Departamento de Ingeniería Mecánica y Mecatrónica. Bogotá D.C., Colombia. E-mail: cjcortesr@unal.edu.co

* Autor de correspondencia: jublancob@sena.edu.co 
experimental design results showed that machining strategy factor on surface roughness parameters $\mathrm{R}_{\mathrm{a}}$. Furthermore, On the other hand, the ANOVA for the concave and convex spherical surfaces shows that the change from concave to convex surface influences geometric tolerance. Finally, ANOVA for convex spherical geometries shows that the radius changes influences surface roughness $\mathrm{R}_{\mathrm{a}}, \mathrm{R}_{\mathrm{q}}$ and $\mathrm{R}_{\mathrm{z}}$, geometric tolerance and the dimensional accuracy.

Keywords: Surfaces sculptured, machining strategy, geometric tolerance.

\section{INTRODUCCIÓN}

El fresado con herramienta de punta esférica de aleaciones de titanio es ampliamente utilizado en las industrias biomédica, aeroespacial y automotriz para la fabricación de piezas que generalmente tienen superficies con diferentes curvaturas, tolerancias y rugosidades que responden a requisitos funcionales y estéticos [1-2]. Estas industrias han mostrado interés en la utilización de este material debido a su elevada relación resistencia/peso. Por esto, en el periodo de los últimos 30 años, la base de datos SCOPUS registra 5027 artículos sobre mecanizado de titanio [3]. De estos, el 2,1\% han tratado sobre fresado de titanio con herramienta de punta esférica [4]. La aleación de titanio comúnmente estudiada es Ti6Al4V (Aleación de Titanio con 6\% de Aluminio y 4\% de Vanadio), la cual representa más del 50\% de la producción de aleaciones de titanio. Cuando esta se mecaniza, ocurre un desgaste rápido debido a la baja conductividad térmica y a la alta temperatura en la zona del filo de corte [5].

En el mecanizado de titanio, como en el de cualquier otro material, es importante la calidad superficial obtenida. En este trabajo, calidad superficial se entiende como el conjunto de características de rugosidad, precisión dimensional y tolerancia de forma. Así, un resultado de interés es la rugosidad $R_{a}$. Este es un valor influenciado por múltiples factores como son los parámetros de mecanizado, las propiedades de la pieza además de la geometría y orientación de la herramienta de corte [6]. En este sentido, diversos estudios concluyen que, al mecanizar una superficie cóncava cilíndrica, el acabado superficial está influenciado por la posición de la herramienta durante el maquinado y el avance de esta [7-9]. En relación a esto, Buj-Corral et al. [10] determinaron que la topografía de la superficie depende del avance por diente, la profundidad de corte radial, la profundidad de corte axial, el número de filos de la herramienta, radios de los filos, ángulo de la hélice, la excentricidad y el ángulo de fase entre los dientes. Adicionalmente, Bağci et al. [11] muestra que hay una influencia de la estrategia y los parámetros de mecanizado sobre la rugosidad en superficies curvas, con los cuales realizan un modelo para predecir esta.

En el fresado de formas complejas, además de los parámetros de corte básicos se debe considerar la estrategia de maquinado. Esta es la disposición del conjunto de trayectorias de la herramienta y la orientación de estas con respecto a la superficie. $\mathrm{La}$ estrategia depende de la topología de la superficie y para su ejecución se requiere una máquina herramienta con configuración de tres o más ejes. Una adecuada selección de la estrategia puede aumentar la eficiencia del maquinado y asegurar la calidad de la superficie. En relación con lo anterior, Lazoglu et al. [12] plantean un estudio de las trayectorias de la herramienta proponiendo la optimización de las fuerzas de corte durante el fresado de superficies de forma libre. Ghorbani et al. [13] presentan ecuaciones para calcular la altura de los scallop (surcos) de superficies cóncavas y convexas basadas en los parámetros que definen la distribución de las trayectorias de la herramienta, lo que contribuye a determinar el acabado superficial.

Además de la rugosidad, se debe tener en cuenta el efecto de las variables del maquinado en las tolerancias dimensionales y geométricas que garantizan el control de la forma de la superficie mecanizada [14]. Así, García et al. [15] plantean un estudio sobre una geometría compleja cóncava, generada a partir de un perfil elíptico, con la cual se generan varios radios de curvatura de manera que se comparan las diferencias en las dimensiones programadas y reales según sea la posición de la curvatura en la superficie; adicionalmente, Wu et al. [16] indican que la estabilidad del fresado disminuye con el aumento en el radio de la curvatura de la superficie del troquel. 
Como se aprecia, aunque se ha investigado bastante sobre el efecto de los parámetros y de la estrategia de maquinado en la calidad superficial de piezas de titanio, se debe estudiar el efecto que provocan el tipo (cóncava o convexa) y radio de la curvatura sobre la rugosidad de la superficie mecanizada, la precisión dimensional y la tolerancia de forma en el fresado con herramienta de punta esférica en máquina herramienta de tres ejes de la aleación Ti6Al4V.

\section{MATERIALES Y MÉTODOS}

La estimación del efecto de los parámetros de corte y la estrategia de maquinado sobre la rugosidad $R_{a}$ (es el valor medio de la rugosidad medida), $R_{q}$ (se define como la raíz cuadrada de la desviación media del perfil medido, o lo que es lo mismo la raíz de la media sobre las irregularidades o imperfecciones que tenemos en la muestra de longitud $\mathrm{L}$ ) y $R_{z}$ (se define como la distancia entre la altura máxima y la altura mínima medida, de las irregularidades o imperfecciones que hemos medido en una longitud $\mathrm{L}$ ), tolerancia geométrica $(T g)$ y precisión dimensional $(P d)$, se hace en tres fases. Mediante un diseño experimental factorial, se estudia el efecto de los parámetros de corte y la estrategia de maquinado. Por otra parte, se usa un análisis de varianza de un factor para estudiar el efecto del sentido de la curvatura (cóncava o convexa) y otro análisis de varianza de un factor para determinar el efecto del radio de curvatura.

Para las diferentes pruebas, se fabricaron probetas cilíndricas de diámetro $7 \mathrm{~mm}$ en cuyos extremos se mecanizaron superficies esféricas convexas con radios de 5; 7,5 y $10 \mathrm{~mm}$ o superficies esféricas cóncavas de radio $10 \mathrm{~mm}$ como se muestran en la Figura 1. Estos radios se seleccionaron con base en la revisión de literatura científica, donde se encontró una prueba de mecanizado en los estos variaban de 0 a $8 \mathrm{~mm}$ en una única superficie cóncava [15] y una prueba de mecanizado de superficies de titanio para una prótesis maxilofacial donde se presentaban múltiples radios y tipos de curvatura donde el menor de los radios correspondía a $2 \mathrm{~mm}$ [17]. Por lo tanto, se decidió analizar una superficie convexa y tomar valores de radio que estuvieran incluidos en la revisión de la literatura $5 \mathrm{~mm}$ y 7,5 mm y un valor de radio que estuviera por fuera del rango analizado, que en este caso fue $10 \mathrm{~mm}$.

Las máquinas, herramientas, materiales, equipos de medición y software empleados aparecen en la Tabla 1. Los factores que se mantuvieron constantes en los diferentes experimentos aparecen en la Tabla 2.

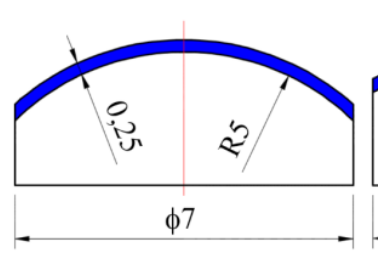

(a)

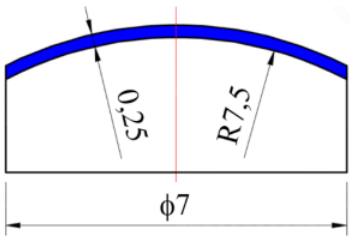

(b)

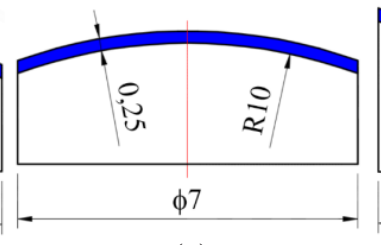

(c)

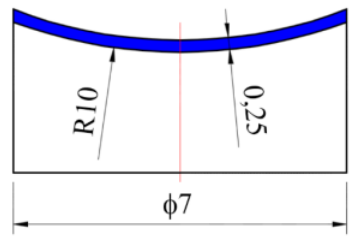

(d)

Figura 1. Dimensiones de las probetas. (a) Superficie convexa $R=5 \mathrm{~mm}$. (b) Superficie convexa $\mathrm{R}=7,5 \mathrm{~mm}$. (c) Superficie convexa $\mathrm{R}=10 \mathrm{~mm}$ (d) Superficie cóncava $\mathrm{R}=10 \mathrm{~mm}$. La línea azul indica el sobrematerial que es maquinado.

Tabla 1. Materiales y equipos usados en las pruebas.

\begin{tabular}{|l|l|l|l|}
\hline \multicolumn{1}{|c|}{ Materiales } & \multicolumn{1}{c|}{ Descripción } & \multicolumn{1}{c|}{ Fabricante } & \multicolumn{1}{c|}{ País } \\
\hline Material maquinado & Titanio Ti6A14V & Baoji Intelle Metals Co. Ltd & China \\
\hline Máquina fresadora & CNC DECKEL FP42NC & Deckel & Alemania \\
\hline Máquina de medición por coordenadas MMC & DuraMax & Zeiss & Alemania \\
\hline Rugosímetro & Infinite Focus G5 & Alicona & Alemania \\
\hline Software CAM & Mastercam & CNC Software, Inc & USA \\
\hline Barra portainserto & KDMB06R150A06HN & Kennametal & USA \\
\hline Inserto de copiado & KDMB06M0ERLD K115M & Kennametal & USA \\
\hline Refrigerante & Swisscool Magnum UX 200 & Motorex AG Langenthal & Suiza \\
\hline
\end{tabular}


Tabla 2. Parámetros constantes en las pruebas experimentales.

\begin{tabular}{|l|c|}
\hline \multicolumn{1}{|c|}{ Parámetro } & Valor $(\mathbf{m m})$ \\
\hline $\begin{array}{l}\text { Sobremedida para maquinado de } \\
\text { superficie esférica convexa o cóncava }\end{array}$ & 0,25 \\
\hline Diámetro de la herramienta & 6 \\
\hline Avance transversal en cada estrategia & 0,25 \\
\hline
\end{tabular}

Tabla 3. Factores y niveles del diseño experimental factorial 23.

\begin{tabular}{|l|c|c|c|}
\hline & $\begin{array}{c}\text { Velocidad } \\
\text { de corte v } \\
(\mathbf{m} / \mathbf{m i n})\end{array}$ & $\begin{array}{c}\text { Avance por } \\
\text { diente f } \\
(\mathbf{m m} / \text { diente })\end{array}$ & $\begin{array}{c}\text { Estrategia } \\
\text { de } \\
\text { maquinado s }\end{array}$ \\
\hline Nivel 1 (-) & $(-) 38$ & $(-) 0,05$ & $(-)$ Paralela \\
\hline Nivel 2 (+) & $(+) 57$ & $(+) 0,10$ & $(+)$ Scallop \\
\hline
\end{tabular}

Mediante un diseño de experimentos factorial $2^{3}$ con 3 réplicas, se analiza el efecto de los parámetros de corte y la estrategia de maquinado en la calidad geométrica. Los niveles de los factores y la designación de cada factor se explican en la Tabla 3 y en la Figura 2. La combinación de factores para cada una las corridas del experimento se muestran en la Tabla 4.

Por otro lado, para observar el efecto del radio de curvatura de la superficie mecanizada se definieron los parámetros de corte que se indican en la Tabla 5 y para cada uno de los tres radios especificados en la Figura 1(a), 1(b) y 1(c) se hicieron 3 probetas. Finalmente, para observar el efecto del sentido de curvatura de la superficie se mecanizaron 3 probetas de superficie convexa y tres de superficie cóncava (Ver Figura 1(a) y 1(d)). En estos casos, el radio de las curvaturas es $10 \mathrm{~mm}$.

\section{RESULTADOS}

\section{Diseño experimental factorial}

Los resultados de las mediciones en el diseño experimental y su designación aparecen en la Tabla 6. El resultado del diseño experimental factorial para la variable $\mathrm{R}_{\mathrm{a}}$ muestra que el parámetro Estrategia de mecanizado es el factor más significativo. Esto se muestra en el diagrama de efectos de Pareto de la Figura 3.

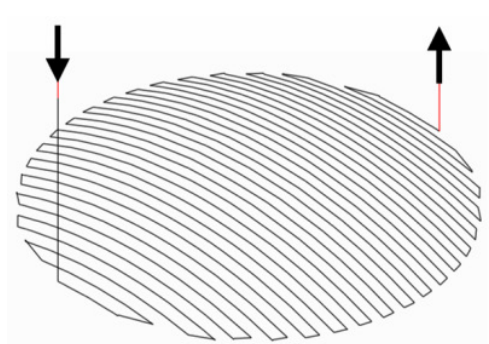

(a)

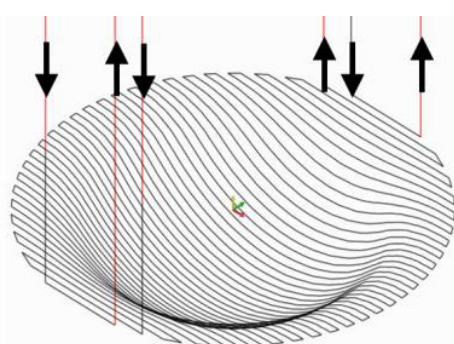

(b)

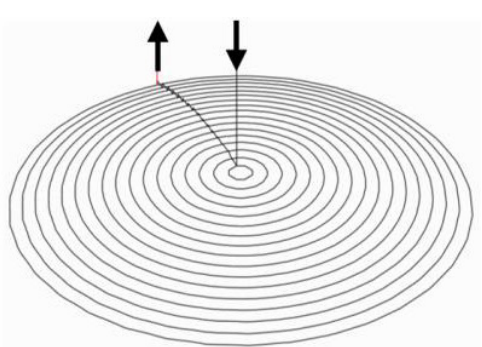

(c)

Figura 2. Descripción gráfica de estrategias de maquinado $(s)$. (a) Estrategia paralela $(s-)$ sobre superficie convexa, (b) Estrategia paralela $(s-)$ sobre superficie cóncava, (c) Estrategia Scallop $(s+)$ sobre superficie convexa.

Tabla 4. Desarrollo del diseño experimental factorial $2^{3}$ con 3 réplicas.

\begin{tabular}{|c|c|c|c|c|}
\hline Orden & Velocidad de corte $\mathbf{v}$ & Avance por diente f & Estrategia de maquinado s & Réplicas \\
\hline 1 & - & - & - & 3 \\
\hline 2 & + & - & - & 3 \\
\hline 3 & - & + & - & 3 \\
\hline 4 & + & + & - & 3 \\
\hline 5 & - & - & + & 3 \\
\hline 6 & + & - & + & 3 \\
\hline 7 & - & + & + & 3 \\
\hline 8 & + & + & + & 3 \\
\hline
\end{tabular}


Tabla 5. Parámetros constantes para el ANOVA de comparación entre curvaturas cóncavas y convexas y el ANOVA de comparación entre radios de curvatura en superficies convexas.

\begin{tabular}{|l|c|}
\hline \multicolumn{1}{|c|}{ Parámetro } & Valor \\
\hline Estrategia de maquinado & Paralela \\
\hline Avance por diente (mm/diente) & 0,05 \\
\hline Velocidad de corte (m/min) & 38 \\
\hline Avance de alimentación (mm/min) & 200 \\
\hline RPM del husillo & 2000 \\
\hline Geometría & Convexa \\
\hline
\end{tabular}

Basado en los resultados del diseño experimental factorial, la ecuación del modelo de regresión estadístico para la rugosidad $R_{a}$ aparece en la ecuación $R_{a}=0,69-0,0014 * s+0,00095 * f+0,53 f * s-$ $0,0138 * v * f * s(1)$ y su representación gráfica se muestra en la Figura 4(a) para la estrategia Paralela.

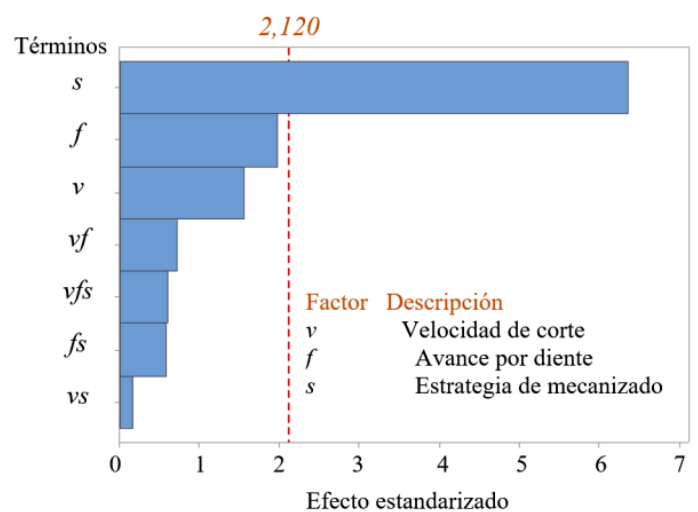

Figura 3. Diagrama de Pareto de efectos estandarizados para la rugosidad $R_{a}$ en el diseño experimental $2^{3}(\alpha=0,05)$.

En la Figura 4(b) para la estrategia Scallop. El porcentaje de variación en la respuesta es explicado por un coeficiente de correlación de $75 \%$.

Tabla 6. Resultados del diseño factorial $2^{3}$.

\begin{tabular}{|c|c|c|c|c|c|}
\hline \multicolumn{3}{|c|}{ Variables de entrada } & \multicolumn{3}{|c|}{ Variables de respuesta } \\
\hline $\begin{array}{c}\text { Velocidad de } \\
\text { corte } v(\mathrm{~m} / \mathrm{min})\end{array}$ & $\begin{array}{c}\text { Avance } \\
f(\mathrm{~mm} / \text { diente) }\end{array}$ & Estrategias & $\begin{array}{c}\text { Rugosidad } \\
\boldsymbol{R}_{a}(\boldsymbol{\mu m})\end{array}$ & $\begin{array}{c}\text { Tolerancia geométrica } \\
\operatorname{Tg}(\mathrm{mm})\end{array}$ & $\begin{array}{l}\text { Precisión dimensional } \\
\text { Pd }(\mathrm{mm})\end{array}$ \\
\hline 38 & 0,05 & Paralela & 0,81 & 0,0113 & 9,93255 \\
\hline 57 & 0,05 & Scallop & 0,80 & 0,0141 & 9,92260 \\
\hline 57 & 0,10 & Scallop & 0,70 & 0,0122 & 9,98605 \\
\hline 38 & 0,05 & Scallop & 0,81 & 0,0140 & 9,93190 \\
\hline 38 & 0,10 & Paralela & 0,67 & 0,0122 & 9,96570 \\
\hline 38 & 0,05 & Paralela & 0,75 & 0,0116 & 10,05750 \\
\hline 57 & 0,05 & Paralela & 0,74 & 0,0185 & 10,16945 \\
\hline 57 & 0,10 & Paralela & 0,69 & 0,0145 & 10,24310 \\
\hline 38 & 0,10 & Scallop & 0,81 & 0,0110 & 10,19090 \\
\hline 57 & 0,05 & Scallop & 0,74 & 0,0119 & 10,17175 \\
\hline 57 & 0,10 & Scallop & 0,74 & 0,0114 & 10,12450 \\
\hline 38 & 0,10 & Scallop & 0,77 & 0,0097 & 10,16670 \\
\hline 57 & 0,05 & Scallop & 0,77 & 0,0123 & 9,93555 \\
\hline 57 & 0,10 & Scallop & 0,65 & 0,0132 & 10,01015 \\
\hline 57 & 0,05 & Paralela & 0,79 & 0,0118 & 9,94235 \\
\hline 38 & 0,10 & Scallop & 0,82 & 0,0117 & 9,96930 \\
\hline 38 & 0,05 & Scallop & 0,69 & 0,0143 & 9,95030 \\
\hline 57 & 0,05 & Paralela & 0,75 & 0,0148 & 9,90465 \\
\hline 57 & 0,10 & Paralela & 0,66 & 0,0170 & 10,19570 \\
\hline 38 & 0,10 & Paralela & 0,87 & 0,0098 & 10,10190 \\
\hline 38 & 0,10 & Paralela & 0,83 & 0,0130 & 10,05620 \\
\hline 57 & 0,10 & Paralela & 0,70 & 0,0125 & 9,91210 \\
\hline 38 & 0,05 & Scallop & 0,62 & 0,0181 & 10,08280 \\
\hline 38 & 0,05 & Paralela & 0,66 & 0,0381 & 10,14820 \\
\hline
\end{tabular}



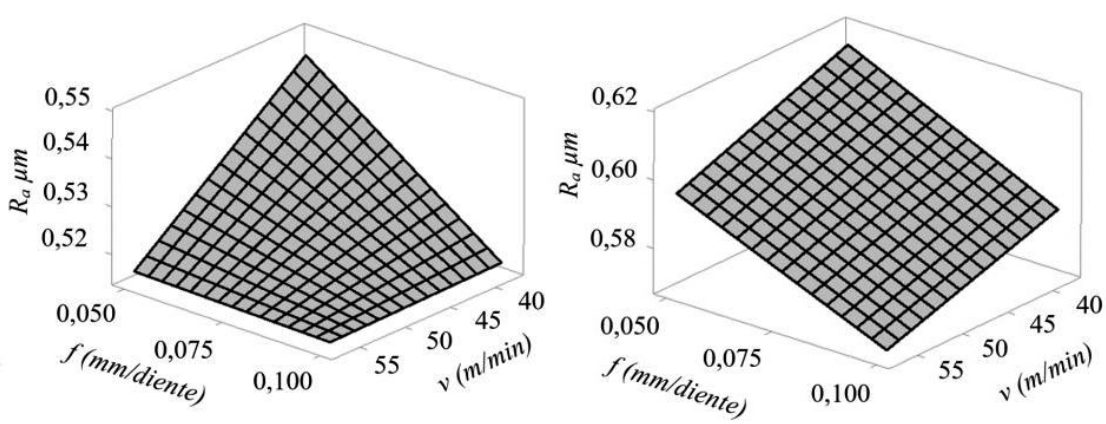

Figura 4. Gráficas de superficie de respuesta de la rugosidad $R_{a}$ en función del avance por diente $f$ y velocidad de corte $v$. (a) Con estrategia paralela $s(-)$, (b) Con estrategia Scallop s (+).

$$
\begin{gathered}
R a=0,69-0,0014 * s+0,00095 * \\
f+0,53 f * s-0,0138 * v * f * s
\end{gathered}
$$

Los valores típicos de cada variable de la ecuación del modelo aparecen en la Tabla 3.

\section{Efecto de curvatura de superficie esférica cóncava y convexa}

El resultado de la medición de los parámetros de la rugosidad superficial, precisión dimensional y tolerancia geométrica para las probetas de superficie esférica cóncava son mostrados en la Tabla 7. Para la variable Tolerancia geométrica $(T g)$ muestra que existe una diferencia significativa entre la superficie cóncava y convexa. La gráfica de valores individuales (Ver Figura 5), muestra que las tolerancias geométricas son más amplias para la superficie cóncava que para la superficie cóncava.

\section{Efecto del radio de curvatura en superficie esférica convexa}

Los resultados de la medición de las variables de la rugosidad $R_{a}, R_{q}$ y $R_{z}$, precisión dimensional y tolerancia geométrica para las probetas de superficie

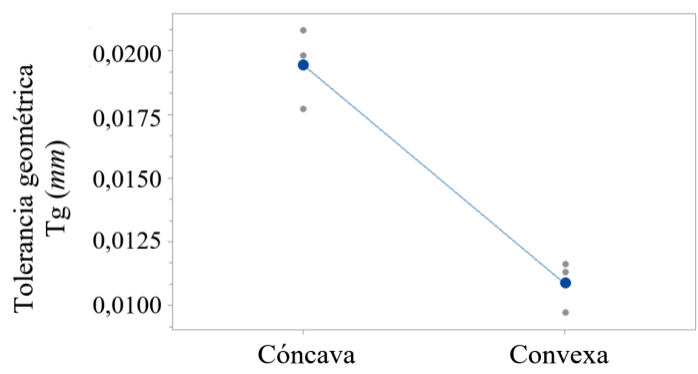

Figura 5. Gráfica valores individuales de Tolerancia geométrica en función del tipo de superficie.

esférica convexa son mostrados en la Tabla 8. Para analizar la precisión dimensional se realizó una normalización de los datos por medio de la ecuación (2), donde $\mathrm{n}$ es el radio normalizado, $r_{m}$ es el radio medido y $r_{0}$ el radio teórico programado.

$$
n=\left|\frac{r_{m}-r_{o}}{r_{0}}\right|
$$

El ANOVA para las variables de respuesta rugosidades $R_{a}, R_{q}$ y $R_{z}$, radio normalizado $(n)$ y tolerancia geométrica $(T g)$ muestra una diferencia significativa

Tabla 7. Resultados del experimento para el ANOVA de comparación entre curvaturas cóncavas y convexas.

\begin{tabular}{|c|c|c|c|}
\hline Variable de entrada & \multicolumn{3}{|c|}{ Variables de respuesta } \\
\hline Tipo de superficie & Rugosidad $\boldsymbol{R}_{\boldsymbol{a}}(\boldsymbol{\mu} \mathbf{m})$ & Tolerancia geométrica $\boldsymbol{T} \mathbf{g}(\mathbf{m m})$ & Precisión dimensional $\boldsymbol{P d}(\mathbf{m m})$ \\
\hline Cóncava & 0,527060 & 0,0198 & 10,00875 \\
\hline Cóncava & 0,521742 & 0,0208 & 9,98935 \\
\hline Cóncava & 0,497656 & 0,0177 & 9,96635 \\
\hline Convexa & 0,556320 & 0,0113 & 9,93255 \\
\hline Convexa & 0,523636 & 0,0116 & 10,0575 \\
\hline Convexa & 0,557304 & 0,0097 & 10,1667 \\
\hline
\end{tabular}


Tabla 8. Resultados del experimento para el ANOVA de comparación entre radios de curvatura en superficies convexas.

\begin{tabular}{|c|c|c|c|c|}
\hline Variable de entrada & \multicolumn{4}{|c|}{ Variables de respuesta } \\
\hline $\begin{array}{c}\text { Radio teórico } \\
\boldsymbol{r}_{\mathbf{0}}(\mathbf{m m})\end{array}$ & $\begin{array}{c}\text { Rugosidad } \\
\boldsymbol{R}_{\boldsymbol{a}}(\boldsymbol{\mu} \mathbf{m})\end{array}$ & $\begin{array}{c}\text { Radio medido } \\
\boldsymbol{r}_{\boldsymbol{m}}(\mathbf{m m})\end{array}$ & $\begin{array}{c}\text { Tolerancia geométrica } \\
\boldsymbol{T} \boldsymbol{(} \mathbf{( m m})\end{array}$ & $\begin{array}{c}\text { Radio normalizado } \\
\boldsymbol{n}(\mathbf{m m} / \mathbf{m m})\end{array}$ \\
\hline 5 & 0,580580 & 4,98630 & 0,0157 & 0,0027400 \\
\hline 5 & 0,620602 & 4,99795 & 0,014 & 0,0004100 \\
\hline 5 & 0,632358 & 4,99690 & 0,0165 & 0,0006200 \\
\hline 7,5 & 0,431284 & 7,52935 & 0,0113 & 0,0039133 \\
\hline 7,5 & 0,485936 & 7,52850 & 0,0150 & 0,0038000 \\
\hline 7,5 & 0,449010 & 7,52005 & 0,0144 & 0,0026733 \\
\hline 10 & 0,556320 & 9,93255 & 0,0113 & 0,0067450 \\
\hline 10 & 0,523636 & 10,05750 & 0,0116 & 0,0057500 \\
\hline 10 & 0,557304 & 10,16670 & 0,0097 & 0,0166700 \\
\hline
\end{tabular}

al variar los radios. Los resultados encontrados muestran que la rugosidad $R_{a}, R_{q}$ y $R_{z}$ presentan una concavidad hacia arriba. Obteniendo su valor de mayor rugosidad para el radio de $5 \mathrm{~mm}$ y el menor para el radio de 7,5 mm. Además, se muestra que el valor de rugosidad para $r=10 \mathrm{~mm}$. no supera un valor de rugosidad para $r r=5 \mathrm{~mm}$ (Ver Figura 6).

En la Figura 7 se muestra que la variación en la precisión dimensional aumenta a medida que aumenta el radio de la superficie. Por otro lado, la Figura 8 muestra que a medida que aumenta el radio disminuye la tolerancia geométrica.

\section{DISCUSIÓN}

\section{Diseño experimental factorial}

En este estudio la gráfica de efectos principales muestra que la estrategia de mecanizado es el factor

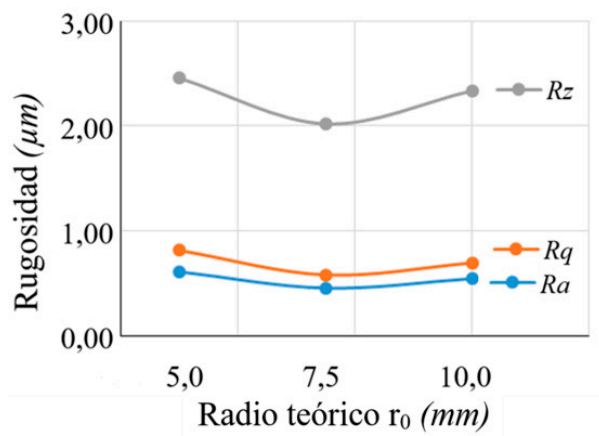

Figura 6. Gráfica de caja de las rugosidades $R_{a}$, $R_{q}, R_{z}$ vs Radios de superficies esféricas convexas.

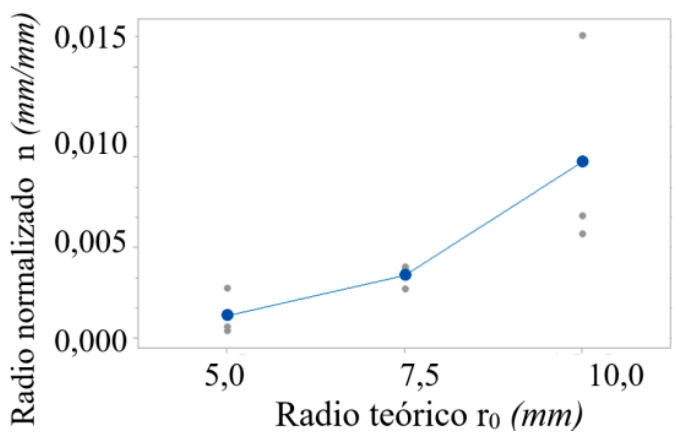

Figura 7. Gráfica de valores individuales para radio normalizado $n$ en función del radio teórico $\mathrm{r}_{0}$ en superficies esféricas convexas.

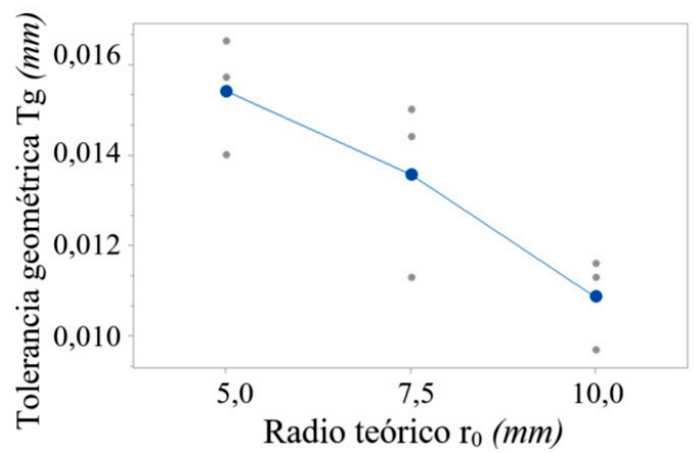

Figura 8. Gráfica de valores individuales para tolerancia geométrica versus Tg en función del radio teórico $\mathrm{r}_{0}$ en superficies esféricas convexas.

que más influye en la rugosidad $R_{a}$. Esto concuerda con Beňo et al. [18] quienes muestran que al variar la estrategia de mecanizado la rugosidad $R_{a}$ cambia. 
Respecto a esto en particular, Mhamdi et al. [19] muestran que al variar la estrategia de mecanizado, la rugosidad de la superficie mecanizada se ve afectada por los efectos termomecánicos generados por las trayectorias del mecanizado que se presentan en el mecanismo de formación de la superficie terminada.

\section{Efecto de curvatura de superficie esférica cóncava y convexa}

En este estudio, la superficie esférica cóncava presenta mayor tolerancia geométrica que la superficie esférica convexa. Estos resultados pueden estar relacionados con la altura del scallop. Sin embargo, los resultados no concuerdan con la teoría, ya que al calcular la altura del scallop por medio de las ecuaciones establecidas por Ghorbani et al. [13] para superficies esféricas cóncavas y convexas, la altura de scallop es menor para la geometría cóncava. La diferencia con respecto a la teoría puede deberse a que el modelo con el que se dedujeron las ecuaciones no corresponde a superficies esféricas como se realizó en este estudio sino a superficies cilíndricas.

Por otra parte, en el estudio de Beňo et al. [18], al comparar el valor experimental de la máxima altura del scallop para una superficie cóncava y convexa de radio $10 \mathrm{~mm}$. muestra que es mayor en la geometría cóncava que en la convexa, lo cual coincide con lo encontrado en este estudio.

\section{Efecto del radio de curvatura en superficie esférica convexa}

Se encontró que la rugosidad $R_{a}, R_{q}$ y $R_{z}$ disminuyen a medida que se incrementa el radio de la superficie esférica convexa, llegan a un mínimo y se incrementan nuevamente. Este resultado es similar al encontrado por Beňo et al. [18]. Por otra parte, la desviación en la precisión dimensional aumenta a medida que aumenta el radio. Esto no concuerda con la ecuación de la superficie convexa de [13] en la cual, se muestra que a medida que se incrementa el radio disminuye la altura del scallop.

Con respecto a la variación de los valores de rugosidad y precisión dimensional, puede deberse al bajo módulo de elasticidad del titanio. Esto ocasiona que en la zona instantánea en la que ocurre el arranque de viruta, se presente deformación y vibración. Por otra parte, se aprecia que la tolerancia geométrica disminuye al incrementar el radio. Este resultado concuerda con la ecuación de Ghorbani et al. [13] para la superficie convexa ya que, al aumentar el radio, disminuye la altura del scallop.

\section{CONCLUSIONES}

Este estudio es relevante debido a que se determinaron los parámetros del fresado que más influyen en la rugosidad superficial y tolerancia geométrica de superficies complejas. Se determinó el efecto de los parámetros de mecanizado, la estrategia de maquinado, el tipo y el radio de curvatura en la calidad geométrica de superficies curvas fresadas en titanio. Se encontró que la estrategia de mecanizado es el factor que más influye en la rugosidad $R_{a}$. Esto se plasma en un modelo de regresión estadístico para la variable de rugosidad $R_{a}$, el cual se muestra en la ecuación 1. También se evidenció que una superficie esférica cóncava, genera una tolerancia geométrica más amplia que una superficie esférica convexa del mismo radio. Por otra parte, al aumentar el radio de una superficie esférica convexa, las variables de rugosidad $R_{a}, R_{q}$ y $R_{z}$ disminuyen hasta un mínimo cuando el radio de curvatura es cercano a 7,5 mm y luego se incrementan nuevamente. El modelo de regresión ofrece la ventaja de predecir la rugosidad superficial $R_{a}$ y la tolerancia geométrica a partir de los valores en el rango de los parámetros evaluados en el fresado y sus limitaciones están en que los resultados aplican para el rango de valores considerados en el experimento.

Los resultados obtenidos pueden emplearse para la fabricación de piezas en industrias como la aeronáutica e implantes médicos, entre otras. Por último, para posteriores estudios es necesario incluir, la evaluación de parámetros como la profundidad de corte, fresado en máquinas de cuatro o más ejes y evaluar el efecto de la geometría del filo de corte.

\section{REFERENCIAS}

[1] K. Jayakumar. "Study of cutting force and surface roughness in Ball nose end milling of vacuum hot pressed A356 alloy/ SiCp metal matrix composite". Materials Today: Proceedings. Vol. 5, pp. 6526-33. 2018. ISSN: 2214-7853. DOI: 10.1016/j. matpr.2017.11.307.

[2] V. Diciuc and M. Lobonţiu. "A review of the main modeling methods for ball nose end 
milling processes". Applied Mechanics and Materials. Vol. 657, pp. 93-7. 2014. ISSN: 1662-7482. DOI: 10.4028/www.scientific. net/AMM.657.93.

[3] B.V. Elsevier. "Machining titanium - Analyze search results". Fecha de consulta: 2020-1109. URL: https://www-scopus-com.bdigital. udistrital.edu.co/term/analyzer.uri?sid=6e ef4d49711fdf5c1be1ff98c78c2d74\&orig in $=$ resultslist $\& s r c=s \& s=T I T L E-A B S-K E$ Y\%28titanium+machining\%29\&sort=plf$\mathrm{f} \& \mathrm{sdt}=\mathrm{b} \&$ sot $=\mathrm{b} \& \mathrm{sl}=33 \&$ count $=10067 \&$ an alyzeResults $=$ Analyze + results $\&$ tx Gid $=c 55$ d630bb7bb2337fda0bbb0a8009566

[4] B.V. Elsevier. "Analyze search results - ball end milling titanium". Fecha de consulta: 2020-11-09. URL. https://www-scopus-com. bdigital.udistrital.edu.co/term/analyzer.uri? sid=2d65c7a19c7555787f149a079ee69787 \&origin $=$ resultslist\&src $=$ s\&s $=A L L \% 28 \mathrm{bal}$ $1+$ end+milling+titanium $\% 29 \&$ sort=plf-f\&s $\mathrm{dt}=\mathrm{b} \&$ sot $=\mathrm{b} \& \mathrm{sl}=30 \&$ count $=2310 \&$ analyze Results $=$ Analyze + results $\&$ txGid $=a 1166 \mathrm{~cd}$ 08fc7079c1eafd6897ee4c605

[5] Z.G. Wang, Y.S. Wong and M. Rahman. "High-speed milling of titanium alloys using binderless CBN tools". International Journal of Machine Tools and Manufacture. Vol. 45, pp. 105-14. 2005. ISSN: 0890-6955. DOI: 10.1016/j.ijmachtools.2004.06.021.

[6] H. Siller, C.A. Rodriguez and H. Ahuett. "Cycle time prediction in high-speed milling operations for sculptured surface finishing". Journal of Materials Processing Technology. Vol. 174, pp. 355-62. 2006. ISSN: 0924-0136. DOI: 10.1016/j.jmatprotec.2006.02.008.

[7] S. Uchikata, A. Beaucamp and Y. Takeuchi. "5-Axis Control Finishing for Decreased Tool Wear". Procedia CIRP. Vol. 63, pp. 313-7. 2017. ISSN: 2212-8271. DOI: 10.1016/j. procir.2017.03.320.

[8] Z.C. Wei, M.L. Guo, M.J. Wang, S.Q. Li and S.X. Liu. "Force predictive model for fiveaxis ball end milling of sculptured surface". The International Journal of Advanced Manufacturing Technology. Vol. 98, pp. 136777. 2018. ISSN: 0268-3768. DOI: 10.1007/ s00170-018-2125-4.

[9] Z.C. Wei, M.L. Guo, M.J. Wang, S.Q. Li and J. Wang. "Prediction of cutting force for ball end mill in sculptured surface based on analytic model of CWE and ICCE". Machining Science and Technology. Vol. 0344. 2019. ISSN: 1532-2483. DOI: 10.1080/10910344.2019.1575408.

[10] I. Buj-Corral, J. Vivancos-Calvet and A. Domínguez-Fernández. "Surface topography in ball-end milling processes as a function of feed per tooth and radial depth of cut". International Journal of Machine Tools and Manufacture. Vol. 53, pp. 151-9. 2012. ISSN: 0890-6955. DOI: 10.1016/j. ijmachtools.2011.10.006.

[11] E. Bağci. "Experimental investigation of effect of tool path strategies and cutting parameters using acoustic signal in complex surface machining". Journal of Vibroengineering. Vol. 19, pp. 5571-88. 2017. ISSN: 13928716. DOI: 10.21595/jve.2017.18475.

[12] I. Lazoglu, C. Manav and Y. Murtezaoglu. "Tool path optimization for free form surface machining". CIRP Annals - Manufacturing Technology. Vol. 58, pp. 101-4. 2009. ISSN: 0007-8506. DOI: 10.1016/j.cirp.2009.03.054.

[13] M. Ghorbani and M.R. Movahhedy. "An analytical model for cutter-workpiece engagement calculation in ball-end finish milling of doubly curved surfaces". International Journal of Advanced Manufacturing Technology. Vol. 102, pp. 1635-57. 2019. ISSN: 1433-3015. DOI: 10.1007/s00170-018-3188-y.

[14] G. Cogorno. "Geometric Dimensioning and Tolerancing for Mechanical Design". McGraw-Hill. 2006.

[15] J.A. García Barbosa, J.M. Arroyo Osorio and E. Córdoba Nieto. "Simulation and verification of parametric numerical control programs using a virtual machine tool". Production Engineering. Vol. 8, pp. 407-13. 2014. ISSN: 1863-7353. DOI: $10.1007 /$ s11740-014-0534-2.

[16] S. Wu, L. Yang, X-li Liu and M-li Zheng. "Effects of curvature characteristics of sculptured surface on chatter stability for die milling". International Journal of Advanced Manufacturing Technology. Vol. 89, pp. 264962. 2017. ISSN: 1433-3015. DOI: 10.1007/ s00170-016-9560-x.

[17] J.A. García Barbosa, J.M. Arroyo Osorio, E. Córdoba Nieto. "Manufacturing of a maxillofacial prosthesis from an axial 
tomography using simulation technologies with a virtual machine tool and four-axis machining". DYNA. Vol. 83, pp. 100-5. 2016. ISSN: $2346-2183$. DOI: $10.15446 /$ dyna.v83n196.49662.

[18] J. Beňo, I. Maňková, P. Ižol and M. Vrabel'. "An approach to the evaluation of multivariate data during ball end milling free-form surface fragments." Measurement: Journal of the
International Measurement Confederation. Vol. 84, pp. 7-20. 2016. ISSN: 02632241. DOI: 10.1016/j.measurement.2016.01.043.

[19] M-B. Mhamdi, M. Boujelbene, E. Bayraktar and A. Zghal. "Surface Integrity of Titanium Alloy Ti-6Al-4V in Ball end Milling". Physics Procedia. Vol. 25, pp. 355-62. 2012. ISSN: $1875-3892$. DOI: $10.1016 / \mathrm{j}$. phpro.2012.03.096. 